\title{
Successful treatment of an extensive verrucous carcinoma with topical 5-aminolevulinic acid-mediated photodynamic therapy
}

\author{
Hsin-Ming Chen ${ }^{1,2}$, Chin-Tin Chen ${ }^{3,4}$, Hsiang Yang ${ }^{1}$, Ming-I Lee ${ }^{5}$, Mark Yen-Ping Kuo ${ }^{1,2}$, \\ Ying-Shiung Kuo ${ }^{1,2}$, Yi-Ping Wang ${ }^{1,2}$, Tsuimin Tsai ${ }^{6} *$, Chun-Pin Chiang ${ }^{1,2, *}$ \\ ${ }^{1}$ Department of Dentistry, National Taiwan University Hospital, Taipei, Taiwan; ${ }^{2}$ School of Dentistry, College of Medicine, National \\ Taiwan University, Taipei, Taiwan; ${ }^{3}$ Graduate Institute of Oral Biology, College of Medicine, National Taiwan University, Taipei, \\ Taiwan; ${ }^{4}$ Center for Optoelectronic Biomedicine, College of Medicine, National Taiwan University, Taipei, Taiwan; ${ }^{5}$ Biomedical \\ Engineering Center, Industrial Technology Research Institute, Hsinchu, Taiwan; ${ }^{6}$ Graduate Institute of Biomedical Materials, Taipei \\ Medical University, Taipei, Taiwan
}

\begin{abstract}
Our recent study found that a new topical 5-aminolevulinic acid-mediated photodynamic therapy (ALA-PDT) protocol composed of multiple 3-min fractionated irradiations with a light emitting diode (LED) red light at $635 \pm 5 \mathrm{~nm}$ for a total of $1000 \mathrm{~s}$ (fluence rate: $100 \mathrm{~mW} / \mathrm{cm}^{2}$; light exposure dose: $100 \mathrm{~J} / \mathrm{cm}^{2}$ ) after topical application of $20 \%$ ALA for 1.5 or $2 \mathrm{~h}$ can be used successfully for the treatment of oral verrucous hyperplasia. In this case report, we tested the efficacy of this new treatment protocol of ALAPDT for an extraoral verrucous carcinoma (VC) lesion at the right mouth angle and an intraoral VC lesion at the right buccal mucosa of a 56-year-old male areca quid chewer and smoker. The extraoral tumor was cleared after six treatments of topical ALA-PDT and the intraoral tumor showed complete regression after 22 treatments of topical ALA-PDT. No recurrence of the VC lesion was found after a follow-up period of 6 months. We suggest that PDT using a topical application of $20 \%$ ALA followed by multiple 3-min fractionated irradiations with an LED red light is also an effective and successful treatment modality for VC.
\end{abstract}

J Oral Pathol Med (2005) 34: 253-6

Keywords: 5-aminolevulinic acid; photodynamic therapy; verrucous carcinoma

*These two authors are equally contributed in this paper.

Correspondence: Dr Chun-Pin Chiang, Department of Dentistry, National Taiwan University Hospital, 1 Chang-Te Street, Taipei 100, Taiwan. Tel.: + 886223 123456x6855. Fax: + 886223 893853. E-mail: cpchiang@ha.mc.ntu.edu.tw

Accepted for publication July 8, 2004

\section{Case report}

This 56-year-old male patient heard from the newspaper that we had successfully treated five patients with oral verrucous hyperplasia $(\mathrm{OVH})$ with topical 5-aminolevulinic acid-mediated photodynamic therapy (ALAPDT). Thus, he came to our outpatient dental clinic and asked for treatment of a verrucous lesion at the right mouth angle and buccal mucosa by ALA-PDT. The extraoral verrucous tumor at the right mouth angle measured about $3 \times 2.5 \times 1 \mathrm{~cm}$ (Fig. 1a); it connected with an intraoral verruous mass which occupied the whole right buccal mucosa and measured about $6 \times 4.5 \mathrm{~cm}$ in area (Fig. 1b). The patient had had areca quid (AQ) chewing (20 quids per day) and smoking habits ( 20 cigarettes per day) since he was 30 years old. He began to notice a verrucous tumor at the right buccal mucosa near the mouth angle 4 years ago. Although he quitted the AQ chewing habit and reduced the consumption of cigarettes to five per day, the tumor continued to grow up to the present form and size. During the past 4 years, the patient had visited the Departments of Oral and Maxillofacial Surgery and of Otorhinolaryngology at five medical centers in the southern and northern part of Taiwan to search for treatment of the lesion. Five incisional biopsies were performed and all the five histopathological reports were a 'benign lesion of OVH' (Fig. 1c). All the oral and maxillofacial surgeons and otorhinolaryngologist advised him to remove the whole lesion by surgery under general anesthesia. Because the patient had atrial fibrillation and was informed by his physician with a life-threatening risk under general anesthesia, he refused to do the surgery. He even refused to do further biopsy, because he insisted that the tumor usually became larger after the intervention of biopsies. Based on the size and appearance of the lesion, the tentative clinical diagnosis was a verrucous carcinoma. 

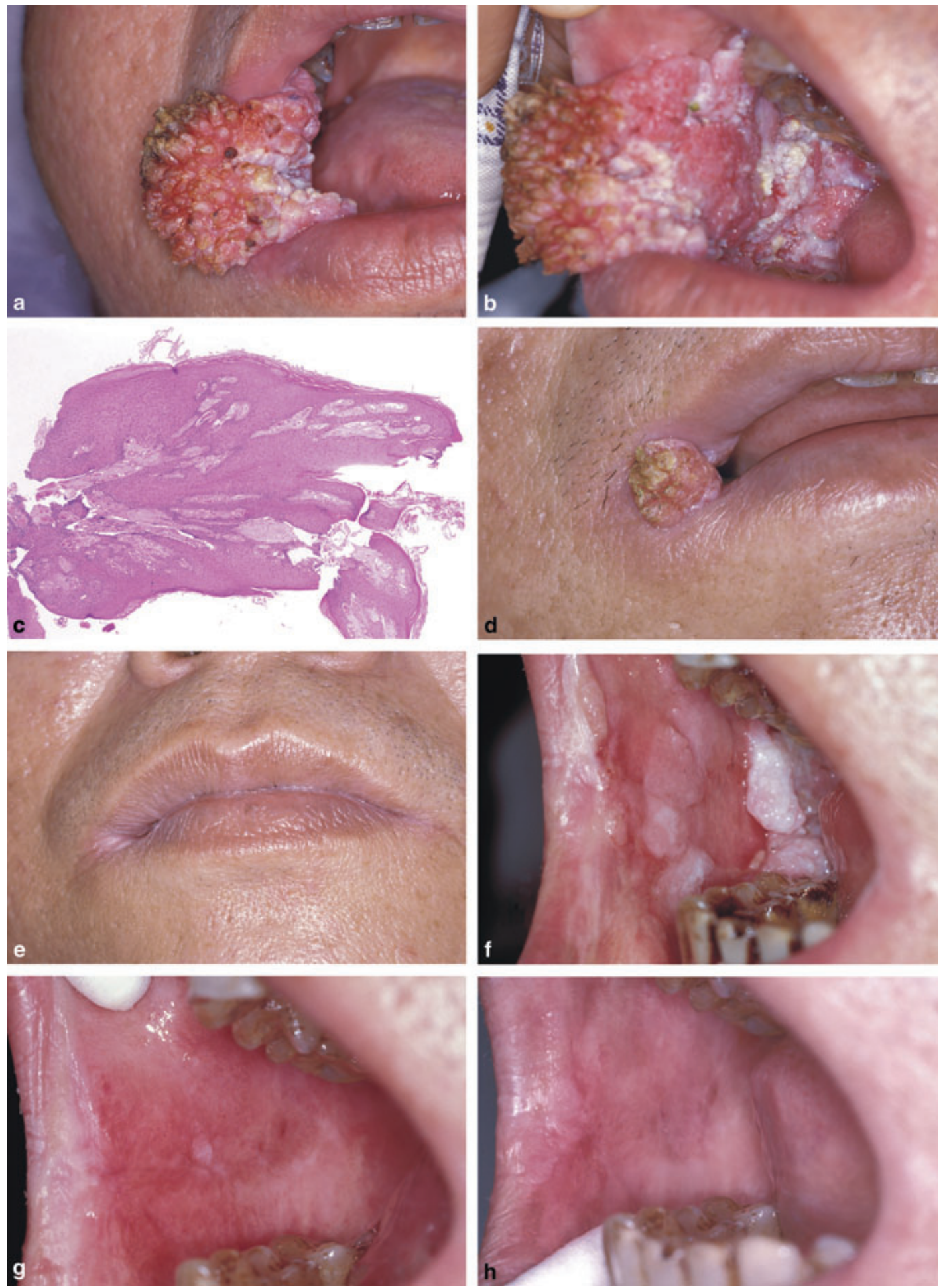

Figure 1 (a) Extraoral verrucous carcinoma (VC) lesion at the right mouth angle before treatment. (b) Extraoral and intraoral VC lesions at the right mouth angle and buccal mucosa before treatment. (c) One previous biopsy of the lesion showing verrucous hyperplasia (hematoxylin and eosin stain; original magnification: $\times 10$ ). (d) Clinical picture of the extraoral VC lesion after three treatments of 5-aminolevulinic acid-mediated photodynamic therapy (ALA-PDT) showing partial regression of the VC lesion. (e) Clinical picture of the patient after six treatments of ALA-PDT showing complete regression of the extraoral VC lesion. (f) Clinical picture of the intraoral VC lesion after 11 treatments of ALA-PDT showing partial regression of the VC lesion. (g) Clinical picture of the right buccal mucosa after 22 treatments of ALA-PDT showing complete regression of the intraoral VC lesion. A small residual white hyperkertotic lesion could be found at the middle portion of the right buccal mucosa and a diffuse white lesion of submucous fibrosis could also be found at the right buccal mucosa, especially the posterior part of the buccal mucosa. (h) Clinical picture of the right buccal mucosa 6 months after completion of ALA-PDT showing no recurrence of the VC lesion. The small residual white hyperkertotic lesion (as seen in g) disappeared spontaneously 6 months later. However, the diffuse white lesion of submucous fibrosis was still present on the right buccal mucosa. 
In view of the recent reports of efficacy of topical ALAPDT, it was decided that a similar approach would be undertaken with the present patient after informed consent was obtained from the patient.

The extraoral tumor at the right mouth angle was treated first by the topical ALA-PDT followed by the intraoral tumor at the right buccal mucosa. The treatment protocol for the present patient was the same as that for the OVH patients as described previously (1). In brief, at the first visit of the treatment, we determined when the protoporphyrine IX (PpIX) reached its peak level in the lesional epithelial cells by ALA-induced PpIX fluorescence spectroscopy. We found that the PpIX reached its maximum level in the lesion $2 \mathrm{~h}$ after local ALA application. Therefore, the subsequent light treatments were set at $2 \mathrm{~h}$ after topical application of ALA to the lesion. The topical ALA-PDT was performed once a week starting from the patient's second appointment of treatment. At the day of treatment, $0.8 \mathrm{ml}$ of $20 \%$ ALA was applied to the extraoral tumor upon patient's arrival. The light treatment composed of multiple 3-min irradiations with a light emitting diode (LED) red light at $635 \pm 5 \mathrm{~nm}$ separated with several 3-min rests for a total of $1000 \mathrm{~s}$ (fluence rate: $100 \mathrm{~mW} / \mathrm{cm}^{2}$; light exposure dose: $100 \mathrm{~J} / \mathrm{cm}^{2}$ ) was delivered $2 \mathrm{~h}$ after topical ALA application. Light treatments were carried out under local anesthesia using $2 \%$ lidocaine with the patient in clear consciousness. The tip of the LED light device was kept as close to the surface of the lesion as possible. Analgesics (acetaminophen, $500 \mathrm{mg} /$ tablet, one tablet four times a day) were prescribed to the patients after PDT treatment. As stated by the patient, this prescription could well control the post-PDT pain. The extraoral tumor was cleared after six treatments of ALAPDT (Fig. 1d,e) and the intraoral tumor showed complete regression after 22 treatments of ALA-PDT (Fig. 1f,g). The patient was then followed up once a week in the first month and once a month thereafter. Because of the systemic condition of atrial fibrillation, the patient refused to do any post-treatment biopsy. No recurrence of the lesion was found after a follow-up period of 6 months (Fig. 1h).

\section{Comments}

In this case report, we treated an extensive verrucous lesion, possibly a verrucous carcinoma (VC), at the right mouth angle and buccal mucosa of a 56-year-old male AQ chewer and smoker by topical ALA-PDT. The large verrucous lesion showed complete regression after a total of 28 treatments of ALA-PDT. Our recent study found that a new topical ALA-PDT protocol composed of multiple 3-min fractionated irradiations with an LED red light at $635 \pm 5 \mathrm{~nm}$ for a total of $1000 \mathrm{~s}$ can be used successfully for the treatment of OVH (1). The result of the present report confirms that this new protocol is also very effective for the treatment of an extensive VC. However, because the effects of AQ and tobacco upon the oral mucosa can last for a further $10-15$ years, a close follow-up is needed to see whether there is a recurrence of the lesion.
ALA itself is not a photosensitizer but serves as the biological precursor of the photosensitizer, PpIX, in the heme biosynthesis pathway. When ALA is topically applied onto the lesion, it diffuses into epithelial cells and then is metabolized into PpIX in the mitochondrial matrix and cytosol. Treatment with a light of $635-\mathrm{nm}$ wavelength can activate PpIX in lesional epithelial cells; PpIX, in turn, transfers energy from light to molecular oxygen, resulting in generation of reactive oxygen species (ROS). There are three possible mechanisms by which ALA-PDT mediates tumor destruction. Firstly, the ROS can kill tumor cells directly. Secondly, PDT can damage the tumor-associated vasculature, leading to thrombus formation and subsequent tumor infarction. Thirdly, PDT can also activate an immune response against tumor cells (1-5).

PDT with systemically or topically administered ALA has been used for the treatment of oral precancerous and cancerous lesions with good clinical outcomes (1-5). Grant et al. (2) first used PDT with orally administered ALA to treat four patients with advanced oral squamous cell carcinoma (SCC). Tumor necrosis was found in three of the four patients after PDT. Fan et al. (3) studied the efficacy of PDT with systemic ALA on 18 oral premalignant and malignant lesions. They found that the depth of tissue necrosis induced by ALA-PDT varied from 0.1 to $1.3 \mathrm{~mm}$, but complete epithelial necrosis was present in all cases. All 12 patients with dysplasia showed regression of the lesion to normal or less dysplastic. Some benefit was observed in five of the six patients with oral SCC after PDT, but only two became tumor-free. PDT with topical ALA can also be used for treating oral leukoplakia (4, 5). Kubler et al. (4) treated 12 oral leukoplakia lesions with PDT after local application of $20 \%$ ALA cream. They observed complete or partial regression in nine oral leukoplakia lesions 3 months later. Sieron et al. (5) treated five oral leukoplakia lesions with PDT after topical exposure of $10 \%$ ALA ointment. Complete response was achieved in four out of five oral leukoplakia lesions. The results of above-mentioned investigations and those of our studies indicate that PDT with either systemic or topical ALA is an effective treatment modality for oral lesions like leukoplakia, $\mathrm{OVH}$ and $\mathrm{VC}$ and at least has some beneficial effects on oral SCCs.

The successful treatment of our VC case with topical ALA-PDT could be at least partially because of our ALA preparation and the new treatment protocol. The $20 \%$ ALA $(\mathrm{w} / \mathrm{w})$ preparation used in this study was a liquid form at room temperature; it became a gel form at body temperature upon contacting the lesional oral mucosa because of a thermoresponsive sol-gel transition of the vehicle (1). The gel form of ALA preparation was adhesive to the oral mucosa and partially resistant to the dilution of the saliva. This characteristic feature of our ALA preparation, in turn, helped the absorption of ALA from the mucosal surface. In addition, the verrucous appearance of the $\mathrm{VC}$ lesion also provided a large area for good retention of ALA on the surface and for good absorption of ALA into cells. In this case report, we divided the 1000 -s light treatment course into 
five periods of $180 \mathrm{~s}$ and a period of $100 \mathrm{~s}$. These six periods of light treatment were interrupted by five periods of 3-min rest. Because an efficient PDT needs sufficient and continuous supply of new PpIX and oxygen, multiple 3-min stops were supposed to give the opportunities for tissues to regenerate new PpIX and to obtain new oxygen. This, in turn, resulted in a successful clinical outcome with this new protocol of treatment.

In this case report, we succeeded in treating an extensive $\mathrm{VC}$ with a new protocol of topical ALA-PDT. Although further studies are needed to verify the real efficacy of this new treatment protocol, we suggest that our new topical ALA-PDT protocol is also an effective and successful treatment modality for VC.

\section{References}

1. Chen HM, Chen CT, Yang H, et al. Successful treatment of oral verrucous hyperplasia with topical 5-aminolevulinic acid-mediated photodynamic therapy. Oral Oncol 2004; 40: 630-7.

2. Grant WE, Hopper C, MacRobert AJ, Speight PM, Bown SG. Photodynamic therapy of oral cancer: photosensitisa- tion with systemic aminolaevulinic acid. Lancet 1993; 342: $147-8$.

3. Fan KF, Hopper C, Speight PM, Buonaccorsi G, MacRobert AJ, Bown SG. Photodynamic therapy using 5-aminolevulinic acid for premalignant and malignant lesions of the oral cavity. Cancer 1996; 78: 1374-83.

4. Kubler A, Haase T, Rheinwald M, Barth T, Muhling J. Treatment of oral leukoplakia by topical application of 5-aminolevulinic acid. Int J Oral Maxillofac Surg 1998; 27: 466-9.

5. Sieron A, Namyslowski G, Misiolek M, Adamek M, Kawczyk-Krupka A. Photodynamic therapy of premalignant lesions and local recurrence of laryngeal and hypopharyngeal cancers. Eur Arch Otorhinolaryngol 2001; 258: $349-52$.

\section{Acknowledgments}

This study was supported by research grants of NSC90-2736-L-002-002 and NSC90-2736-L-002-003 from the National Science Council, Taipei, Taiwan. The authors thank Industrial Technology Research Institute of Taiwan for the development of the light emitting diode source. 